\title{
The effect of gravity on R410A condensing flow in horizontal circular tubes
}

\author{
Wei Li, Jingzhi Zhang, Pengfei Mi, Jianfu Zhao, Zhi Tao, Peter R. N. Childs \& \\ Tom I.-P. Shih
}

To cite this article: Wei Li, Jingzhi Zhang, Pengfei Mi, Jianfu Zhao, Zhi Tao, Peter R. N. Childs \& Tom I.-P. Shih (2017) The effect of gravity on R410A condensing flow in horizontal circular tubes, Numerical Heat Transfer, Part A: Applications, 71:3, 327-340, DOI: 10.1080/10407782.2016.1264743

To link to this article: http://dx.doi.org/10.1080/10407782.2016.1264743

\section{曲 Published online: 13 Feb 2017.}

Submit your article to this journal

山 Article views: 71

Q View related articles ¿ك

View Crossmark data $₫$ 


\title{
The effect of gravity on R410A condensing flow in horizontal circular tubes
}

\author{
Wei Li ${ }^{\mathrm{a}}$, Jingzhi Zhang ${ }^{\mathrm{b}, \mathrm{c}}$, Pengfei $\mathrm{Mi}^{\mathrm{b}}$, Jianfu Zhao ${ }^{\mathrm{d}}$, Zhi Tao ${ }^{\mathrm{e}}$, Peter R. N. Childs ${ }^{\mathrm{b}}$, and \\ Tom I.-P. Shih ${ }^{f}$ \\ aDepartment of Energy Engineering, Zhejiang University, Hangzhou, Zhejiang, China; byson School of Design \\ Engineering, Imperial College London, London, UK; 'Co-Innovation Center for Advanced Aero-Engine, Zhejiang

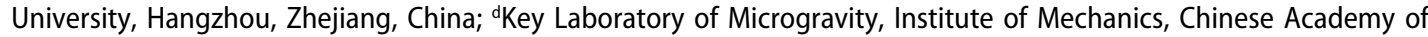 \\ Sciences, Beijing, China; 'School of Energy and Power Engineering, Beijing University of Aeronautics and Astronautics, \\ Beijing, China; 'School of Aeronautics and Astronautics, Purdue University, West Lafayette, Indiana, USA
}

\begin{abstract}
Heat transfer characteristics of R410A condensation in horizontal tubes with the inner diameter of $3.78 \mathrm{~mm}$ under normal and reduced gravity are investigated numerically. The results indicate that the heat transfer coefficients increase with increasing gravitational accelerations at a lower mass flux, whereas their differences under varying gravity are insignificant at a higher mass flux. The liquid film thickness decreases with increasing gravity at the top part of the tube, whereas the average liquid film thickness is nearly the same under different gravity accelerations at the same vapor quality and mass flux. The local heat transfer coefficients increase with increasing gravity at the top of the tube and decrease with increasing gravity at the bottom. The proportion of the thin liquid film region is important for the overall heat transfer coefficients for the condensing flow. A vortex with its core lying at the bottom of the tube is observed under normal gravity because of the combined effect of gravity and the mass sink at the liquid-vapor interface, whereas the stream traces point to the liquid-vapor interfaces under zero gravity. The mass transfer rate under zero gravity is much lower than that of normal gravity.
\end{abstract}

\section{ARTICLE HISTORY}

Received 10 January 2016

Accepted 4 November 2016

\section{Introduction}

Compared with single-phase flows, two-phase flows can leverage the latent heat rather than the sensible heat alone and achieve heat transfer enhancement. These flows have the potential to meet the increasing demand of high heat dissipation in thermal management systems for electronic equipment, energy systems, and space missions. With the growing interest in space applications and power requirements of the equipment on space vehicles, more studies are focused on the two-phase flow such as pool boiling, flow boiling, and condensation under reduced and microgravity conditions.

The gravity effect is reflected in buoyancy, which is determined by gravity and the density difference between liquid and vapor. Gravity can play a crucial role in the bubble detachment and the critical heat flux (CHF) in pool and flow boiling, and it also influences the flow patterns in flow boiling and condensation. Numerous experimental and numerical investigations have been conducted on the gravity effect on nucleate and film pool boiling [1-4]. Compared with pool boiling, flow boiling could enhance CHF in microgravity because the liquid motion could take the bubbles away

CONTACT Wei Li $\bigotimes$ weili96@zju.edu.cn Department of Energy Engineering, Zhejiang University, 38 Zheda Road, Hangzhou, 310027, Zhejiang, P.R. China.

Color versions of one or more figures in the article can be found online at www.tandfonline.com/unht. 


\section{Nomenclature}

\begin{tabular}{|c|c|}
\hline$A$ & area, $\mathrm{m}^{2}$ \\
\hline$c_{\mathrm{p}}$ & specific heat capacity, $\mathrm{J} \mathrm{kg}^{-1} \mathrm{~K}^{-1}$ \\
\hline$d_{\mathrm{h}}$ & hydraulic diameter, $\mathrm{m}$ \\
\hline$E$ & specific sensible enthalpy, $\mathrm{J} \mathrm{kg}^{-1}$ \\
\hline$F$ & surface tension force, $\mathrm{N} \mathrm{m}^{-3}$ \\
\hline$g$ & gravitational acceleration, $\mathrm{m} \mathrm{s}^{-2}$ \\
\hline G & mass flux, $\mathrm{kg} \mathrm{m}^{-2} \mathrm{~s}^{-1}$ \\
\hline$h$ & heat transfer coefficient, $\mathrm{W} \mathrm{m}{ }^{-2} \mathrm{~K}^{-1}$ \\
\hline$h_{l v}$ & latent heat of vaporization, $\mathrm{J} \mathrm{kg}^{-1}$ \\
\hline$k$ & thermal conductivity, $\mathrm{W} \mathrm{m}{ }^{-1} \mathrm{~K}^{-1}$ \\
\hline$m$ & mass source due to phase change, $\mathrm{kg} \mathrm{m}^{-3} \mathrm{~s}^{-1}$ \\
\hline MAD & mean average deviation, $[-]$ \\
\hline MRD & mean relative deviation, $[-]$ \\
\hline$r$ & coefficient of mass source, $\mathrm{s}^{-1}$ \\
\hline$P r_{t}$ & turbulent Prandtl number, - \\
\hline$T$ & temperature, $\mathrm{K}$ \\
\hline
\end{tabular}

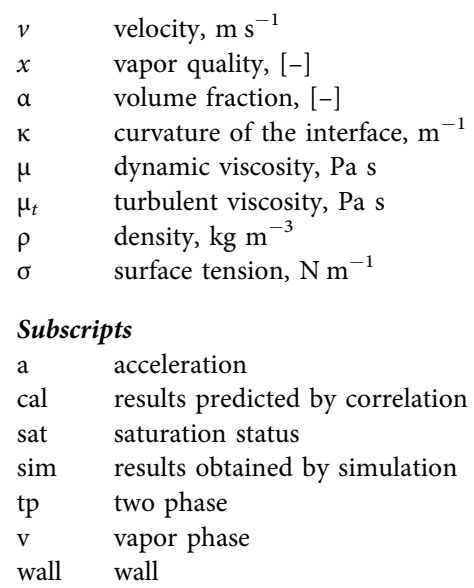

and replenish the wall. Konishi and Mudawar [5] made a thorough review about the flow boiling and $\mathrm{CHF}$ in microgravity discussing several models for predicting CHF and highlighting the need for further research emphasis on condensation flow and heat transfer characteristics under reduced gravity.

Compared with flow boiling and nucleate boiling investigations, research works on condensation under microgravity were limited. Lee et al. [6] experimentally investigated the condensing flow of FC-72 under microgravity. The reduced gravity environment was achieved by parabolic flights. At lower mass fluxes, the condensation heat transfer coefficient was determined by the thermal conduction in the liquid film. The combined effects of turbulence and interfacial waviness were found to enhance heat transfer downstream at higher mass fluxes. The empirical correlations based on the normal gravity were found to reasonably predict the microgravity data. They [7] also conducted condensation experiments under microgravity, lunar gravity, and Martian gravity. The results showed that gravity effects were pronounced at lower mass flux. Bortolin et al. [8] reported four experiments conducted under reduced gravity, including condensation on external surfaces, falling films condensation, in-tube condensation of n-pentane, and convective condensation of R134a in a square channel.

Other experimental works relating to the gravity effect on condensation have been mainly conducted in inclined tubes. By changing the tube inclination, the gravity component along the radial direction decreases. Lips and Meyer [9] undertook a review on two-phase condensation flow in inclined tubes, including flow pattern maps, void fractions, heat transfer coefficients, and pressure drops. The inclination angle affected the flow patterns, leading to a significant influence on the heat transfer coefficients and pressure drops. An optimum inclination angle could be found for a specific configuration. Akhavan-Behabadi et al. [10] carried out an experimental work of R134a condensation in a microfin tube with an inner diameter of $8.92 \mathrm{~mm}$. The results indicated that the highest heat transfer coefficients were obtained when the inclination angle was $30^{\circ}$. The effects of inclination angles on the heat transfer coefficients were more pronounced at lower mass flux, which is similar to the conclusion of Lee et al. [7]. The flow patterns of condensing flow in microfin tubes were presented in Mohseni and Akhavan-Behabadi [11]. Lips and Meyer [12,13] investigated the effects of inclination angles on the convective condensation for R134a in a 8.38-mm smooth tube at mass fluxes ranging from 200 to $600 \mathrm{~kg} \mathrm{~m}^{-2} \mathrm{~s}^{-1}$ and vapor quality ranging from 0.1 to 0.9 . The highest heat transfer coefficient was found at an inclination angle of $-15^{\circ}$, whereas the heat transfer was deteriorated for the upward flow. Del Col et al. [14] experimentally studied the condensation processes in mini square channels for R134a and R32 at a saturation temperature of $313.15 \mathrm{~K}$ and mass fluxes between 100 and $390 \mathrm{~kg} \mathrm{~m}^{-2} \mathrm{~s}^{-1}$. A correlation to determine the critical mass flux at which the inclination angle starts to influence the heat transfer of condensation was proposed. 
Compared with the experimental studies, numerical work on condensing flow is limited. For the numerical studies relating to the multiphase flow, the volume-of-method (VOF) method and the Level-set method have been widely adopted to track the liquid-vapor interfaces. The transient and steady numerical simulations performed have been mainly focused on different aspects. The former method was adopted to study the interfacial waviness, the intermittent flow patterns like bubbly/slug flow, or the transitions of the flow pattern regimes. The studies using steady simulations have mainly focused on the steady liquid film thicknesses, the liquid-vapor interfaces, and the local heat transfer coefficients. Ganapathy et al. [15] studied the transient condensing flow in micro channels $\left(d_{\mathrm{h}}=100\right.$ $\mu \mathrm{m})$ using two-dimensional models. The numerical flow patterns, heat transfer coefficient, and pressure drops agreed well with other studies in the open literature. The effect of the wall heat flux on the heat transfer coefficients was less important than that of the mass flux. Lee et al. [16] conducted experimental and computational investigations of vertical down-flow condensation. The computed heat transfer coefficient agreed well with the experimental results. Chen et al. [17] performed a three-dimensional simulation of FC-72 in a rectangular microchannel with a 1-mm hydraulic diameter. Although the cross-sectional meshes were coarse, the numerical work captured the transition from the annular flow to the bubbly/slug flow and presented some vectors and temperature distributions for these flow patterns. Other numerical studies were mainly performed using the steady model in which liquid film waviness and intermittent flow patterns could not be predicted. Wang and Rose [18-23] made significant contributions to the condensation numerical work using the laminar flow assumption for the liquid film. The effects of surface tension, gravity, and tube shapes on condensation heat transfer coefficients were explored in detail. Compared with surface tension, gravity is unnoticeable in the noncircular channels. However, most of the numerical results showed that the heat transfer coefficient was not dependent on the mass flux. Da Riva et al. [24-26] adopted the low-Reynolds SST $k \sim \omega$ model to consider the turbulence effect in the liquid film and obtained a good agreement between the numerical results and the corresponding experimental data. Based on this approach, Bortolin et al. [27] and Zhang et al. [28] studied the effect of channels shapes (square and flattened) and Zhang et al. [29] investigated the effect of saturation temperatures on the condensing flow.

As most of the condensation work was carried out at normal gravity, the effect of gravity on the condensing flow has not been fully studied. Such work will aid the designer in producing more efficient and reliable thermal control systems for space vehicles or other equipment under reduced and microgravity. Former numerical works relating to condensation under microgravity mainly used R134a as the working fluid [24], whereas the simulations using R410A are limited. Obviously, different fluid properties will lead to different conclusions. The present work mainly focuses on the effects of gravity on the heat transfer characteristics for R410A condensing flow inside circular smooth tubes with an inner diameter of $3.78 \mathrm{~mm}$. The numerical local heat transfer coefficients are also compared with widely used correlations to validate the numerical method adopted in this work. The local heat transfer coefficients, liquid film thickness, and stream traces are presented to give a better understanding of the gravity effect on condensation.

\section{Numerical model}

The numerical simulation was conducted using ANSYS Fluent (Release 14.5) with the VOF model to capture the liquid-vapor interfaces. Detailed descriptions of the numerical model, numerical discretization methods, and boundary conditions can be found in our previous work [29]. Here, only a brief introduction of this method is given.

\subsection{Governing equations}

Turbulent effects in the liquid and vapor flows during the condensation process are considered as recommended by Da Riva et al. [26], using the SST k $\sim \omega$ turbulent model. The same approach was 
also adopted in Bortolin et al. [27], Kharangate et al. [30], and Lee et al. [16]. The basic equations for the continuity, momentum, energy, turbulence kinetic energy, and the specific dissipation rate are the same as those of single-phase flow as listed in Eq. (1)-Eq. (3). A volume fraction equation is coupled with these equations to distinguish the two phases. The properties used in these equations are the volume-fraction-weighted values of liquid and vapor.

A continuum surface force (CSF) model [31] is used to consider the surface tension effect, which is of importance for two-phase flow in mini/microchannels. The phase-change phenomenon is modeled by adding source terms in the volume fraction equation and the energy equation using the userdefined functions (UDF) in Ansys Fluent. As all these equations are coupled, the effect of the source terms in the volume fraction and energy equations will also influence the velocity and pressure distributions.

Continuity Equation:

$$
\nabla \cdot(\rho \vec{v})=0
$$

Momentum Equation:

$$
\nabla \cdot(\rho \vec{v} \vec{v})=-\nabla P+\nabla \cdot\left[\left(\mu+\mu_{t}\right)\left(\nabla \vec{v}+\nabla_{\vec{v}}^{T}\right)\right]+\rho \vec{g}+\vec{F}
$$

Energy Equation:

$$
\nabla \cdot[\vec{v}(\rho E+P)]=\nabla \cdot[k(\nabla T)]+h_{l v} m_{l}
$$

Volume Fraction Equation:

$$
\vec{v} \cdot \nabla \alpha_{i}=\frac{m_{i}}{\rho_{i}}
$$

Turbulence Kinetic Energy Equation:

$$
\frac{\partial}{\partial t}(\rho k)+\frac{\partial}{\partial x_{i}}\left(\rho k v_{i}\right)=\frac{\partial}{\partial x_{j}}\left(\Gamma_{k} \frac{\partial k}{\partial x_{i}}\right)+G_{k}^{\prime}-Y_{k}+S_{k}
$$

Specific Dissipation Rate Equation:

$$
\frac{\partial}{\partial t}(\rho \omega)+\frac{\partial}{\partial x_{i}}\left(\rho \omega v_{i}\right)=\frac{\partial}{\partial x_{j}}\left(\Gamma_{\omega} \frac{\partial \omega}{\partial x_{i}}\right)+G_{\omega}^{\prime}-Y_{\omega}+S_{\omega}+D_{\omega}
$$

where:

$$
\begin{gathered}
\alpha_{l}+\alpha_{v}=1 \\
\vec{F}=\frac{2 \sigma \rho \kappa_{l} \nabla \alpha_{l}}{\rho_{l}+\rho_{v}} .
\end{gathered}
$$

More details about the definitions of the parameters in these equations can be found in Zhang et al. [29] and the Ansys Fluent manuals.

The source terms for the mass transfer in Eq. (3) and Eq. (4) are based on the Lee [32] model, which is widely adopted for condensation [16,17,25-27,29,30] and evaporation [33,34] simulations. As shown in Eq. (9), when the temperature of the vapor phase in a computational cell is lower than the saturation temperature, a negative mass source term is expected for the vapor phase corresponding to the condensation process, and vice versa for the evaporation phenomena. The volume fraction of the liquid or vapor phase is adopted in the mass transfer model, which ensures that the mass transfer only occurs near the liquid-vapor interface where the volume fractions are between 0 and 1. In the cases of lower liquid temperature or higher vapor temperature than the saturation temperature, the mass term transferring through the liquid-vapor interface is zero [29].

In the Lee [32] model, a coefficient $r$ is adopted, which depends on the particular cases under study. For the evaporation and boiling simulations, a smaller $r$ is usually applied, whereas a much 


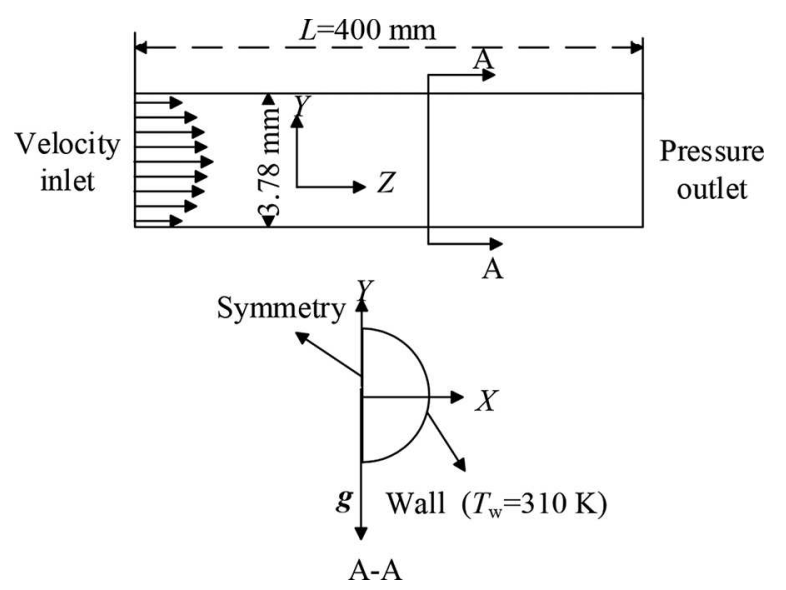

Figure 1. Computational geometry and boundary conditions.

higher $r$ is adopted in the condensation numerical work. The values of $r$ for the condensation simulations range from $5,000 \mathrm{~s}^{-1}$ in Liu et al. [35] to $5 \times 10^{6} \mathrm{~s}^{-1}$ in Da Riva et al. [26] to keep the interface temperature within $T_{\text {sat }} \pm 1 \mathrm{~K}$. In the present work, $r=1.5 \times 10^{6} \mathrm{~s}^{-1}$ is adopted, for which the difference between the calculated liquid-vapor interface temperature and saturation temperature is lower than $0.5 \mathrm{~K}$.

$$
\begin{gathered}
m_{v}=\left\{\begin{array}{cl}
r \rho_{v} a_{v} \frac{T-T_{\text {sat }}}{T_{\text {sat }}}, & \text { if } T \leq T_{\text {sat }} \\
r \rho_{l} a_{l} \frac{T-T_{\text {sat }}}{T_{\text {sat }}}, & \text { if } T>T_{\text {sat }}
\end{array}\right. \\
m_{l}=-m_{v}
\end{gathered}
$$

\subsection{Computational domain}

Figure 1 shows the computational domain for a horizontal smooth tube studied in the present work. A velocity inlet boundary condition is applied at the tube inlet with a constant temperature $\left(T_{\text {sat }}=320 \mathrm{~K}\right)$, turbulent kinetic energy, and the turbulent specific dissipation rate. A pressure outlet boundary condition is adopted at the downstream boundaries to avoid reverse flow. A symmetry boundary condition is applied on the $Y Z$ plane. The normal gradients for all solved variables are zero on this plane. A no-slip boundary condition with a constant temperature $\left(T_{\mathrm{w}}=310 \mathrm{~K}\right)$ is used as the wall boundary condition. The properties of R410A used in the present work are obtained from REFPROP 8.0. The gravity effect is considered by the gravitational acceleration along the $Y$-axis as shown in Figure 1.

The values for inlet velocity together with the turbulent kinetic energy and the turbulent specific dissipation rate are obtained by periodic single-phase simulations. After obtaining the distributions of the fully developed single-phase velocity, turbulent kinetic energy, and turbulent specific dissipation rate, a profile file is used to convert these values into the condensation simulations. The length of the single-phase computational model is $10 d_{h}$ in length, and the cross-sectional mesh distribution is the same as that of the two-phase numerical domain. Periodic boundary conditions are applied at the tube inlet and the outlet. The velocity components repeat themselves in space with a constant pressure drop between modules. The equations of the periodic boundary conditions are shown below:

$$
\begin{gathered}
\vec{v}(z)=\vec{v}(z+L)=\vec{v}(z+2 L)=\ldots \\
\Delta P=P(z)-P(z+L)=P(z+L)-P(z+2 L)=\ldots
\end{gathered}
$$




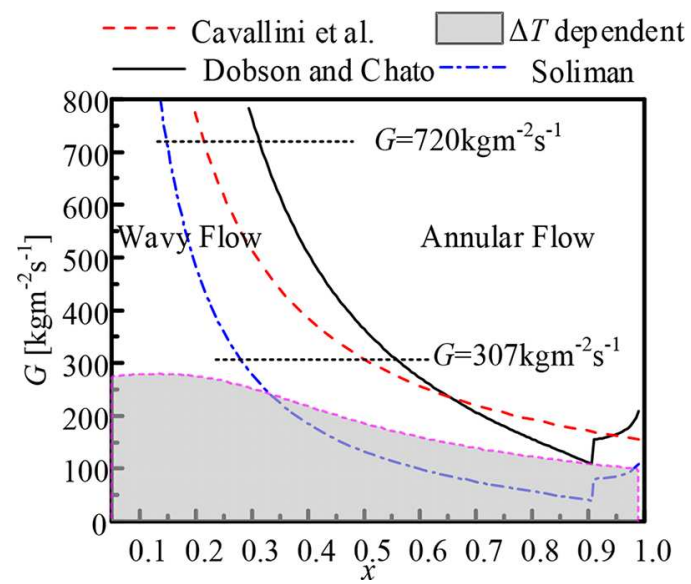

Figure 2. Flow pattern maps proposed by Dobson and Chato [36], Cavallini et al. [37], Soliman [38], and Cavallini et al. [39].

The hexahedral cells with a fine boundary layer mesh near the wall region is used in the present work, whereas much coarser meshes are adopted in the core region where the vapor phase dominates. An enhanced wall treatment, which is the default for all $\omega$-equation based turbulent models in Ansys Fluent, is applied to solve the viscous sublayer and the logarithmic layer near the tube wall where a liquid film exists. Similar to Da Riva et al. [26], a mesh sensitivity study is conducted under Earth's gravity in terms of the average heat transfer coefficients at the wall. The average heat transfer coefficients' difference between the fine mesh (around 2.66 million elements) and the coarse mesh (around 1.35 million elements) is less than $0.6 \%$. In this study, the coarse mesh is used.

\section{Results and discussions}

\subsection{Flow patterns and liquid-vapor interfacial temperatures}

Figure 2 shows the flow pattern maps proposed by Dobson and Chato [36], Cavallini et al. [37], Soliman [38], and Cavallini et al. [39]. As shown in Figure 2, annular flow occurs for the mass flux $G=307 \mathrm{~kg} \mathrm{~m}^{-2} \mathrm{~s}^{-1}$ and $G=720 \mathrm{~kg} \mathrm{~m}^{-2} \mathrm{~s}^{-1}$ at vapor quality ranging from 0.45 to 0.95 , and all the cases studied belong to the $\Delta T$-independent region. The range of the annular flow regime extends with decreasing gravity. As the steady-state simulation cannot predict the wavy and slug/bubbly flow regimes, the effect of fluctuation on the liquid-vapor interface is ignored in the present study.

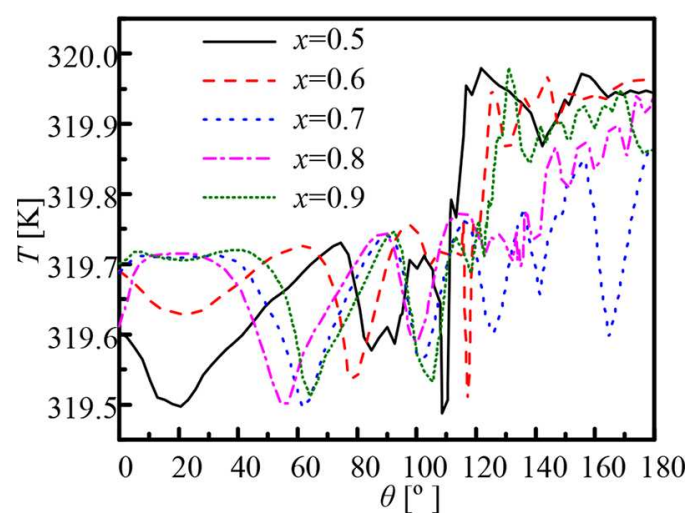

Figure 3. Temperatures of the liquid-vapor interfaces. 


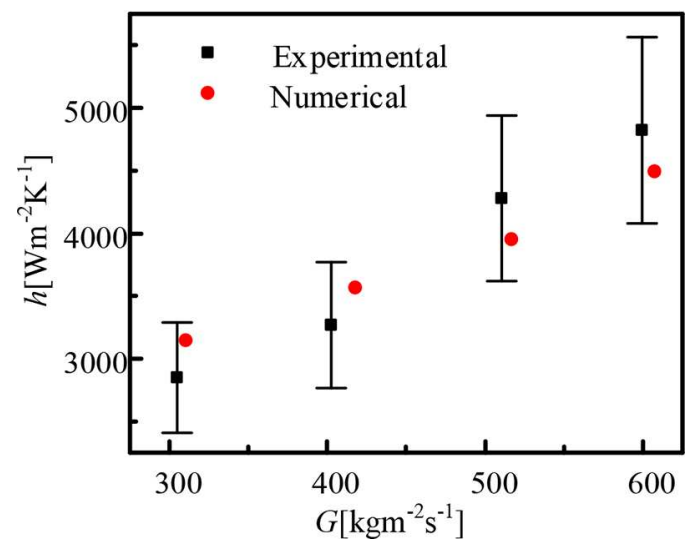

Figure 4. Comparison of numerical data with the experimental results at $x=0.45$.

As mentioned before, a constant temperature is assumed at the liquid-vapor interface in the Lee [32] model. However, the liquid-vapor interfacial temperature varies during the computational process. A rational $r$ is chosen to keep the computational interfacial temperature within a small range of the refrigerant saturation temperature. Figure 3 shows the interfacial temperature at $x$ ranging from 0.5 to 0.9 plotted against the angular coordinate moving from the tube top to the bottom. For the $r$ adopted in the present work, the interfacial temperatures range from 319.5 to $319.9 \mathrm{~K}$, which indicates that $r=1.5 \times 10^{6} \mathrm{~s}^{-1}$ is big enough to perform the Lee [32] model.

\subsection{Comparison of the numerical data with the experimental results and empirical correlations under normal gravity}

The heat transfer coefficients for the condensing flow obtained using the models described before are compared with the experimental data in our previous experimental studies [40]. More details about the experimental setup and data reduction can be found in $\mathrm{Wu}$ et al. [40]. Figure 4 shows the comparison between the numerical heat transfer coefficient obtained by the average local heat transfer coefficient at a local vapor quality and the experimental results. The numerical heat transfer coefficient increases with increasing mass flux and falls into the relative accuracy $( \pm 15.4 \%)$ of the experimental setup.

$$
h=\frac{1}{A} \int \frac{q_{\text {wall }}}{T_{\text {sat }}-T_{\text {wall }}} d A
$$

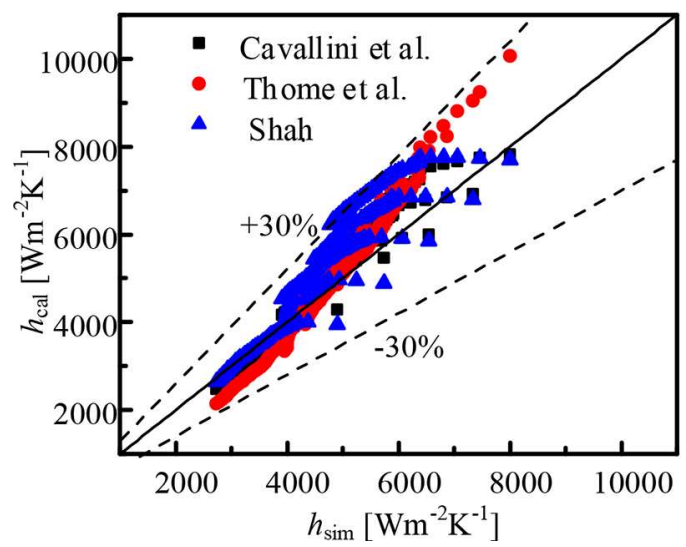

Figure 5. Comparison with the empirical correlations for heat transfer coefficients. 
Table 1. MAD and MRD calculated by different correlations.

\begin{tabular}{lcr}
\hline Correlations & MAD (\%) & MRD (\%) \\
\hline Cavallini et al. [39] & 8.9 & 5.7 \\
Thome et al. [41] & 9.3 & 1.1 \\
Shah [42] & 13.0 & 11.7 \\
\hline
\end{tabular}

The local numerical heat transfer coefficients are also compared with the empirical correlations developed by Cavallini et al. [39], Thome et al. [41], and Shah [42]. As shown in Figure 5, the heat transfer correlations can predict the numerical data well. The mean absolute deviation (MAD) and the mean relative deviation (MRD) for these correlations, which are calculated in Eq. (14) and Eq. (15), respectively, are listed in Table 1. The maximum MAD for these three correlations is about $13.0 \%$, which validates the Lee [32] model and the numerical method used in the present work. In the following work, the same heat and mass transfer model is adopted in the numerical studies of R410A condensation under reduced and microgravities.

$$
\begin{gathered}
\mathrm{MAD}=\frac{1}{N} \sum_{1}^{N}\left|\frac{U_{\text {pre }}-U_{\text {sim }}}{U_{\text {sim }}}\right| \bullet 100 \% \\
\mathrm{MRD}=\frac{1}{N} \sum_{1}^{N} \frac{U_{\text {pre }}-U_{\text {sim }}}{U_{\text {sim }}} \bullet 100 \%
\end{gathered}
$$

where $U$ is the value of a numerical or calculated data point.

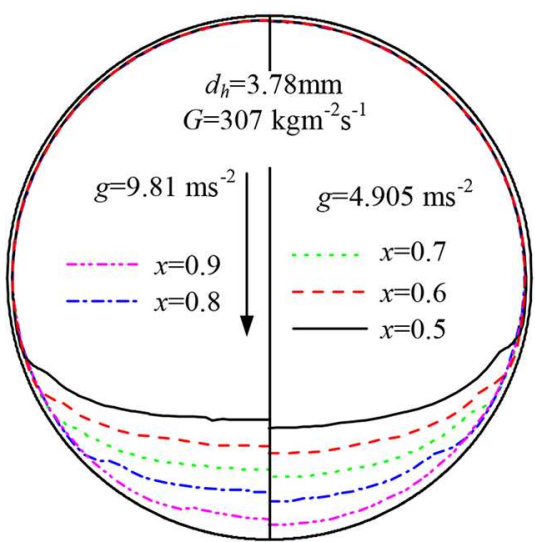

(a)

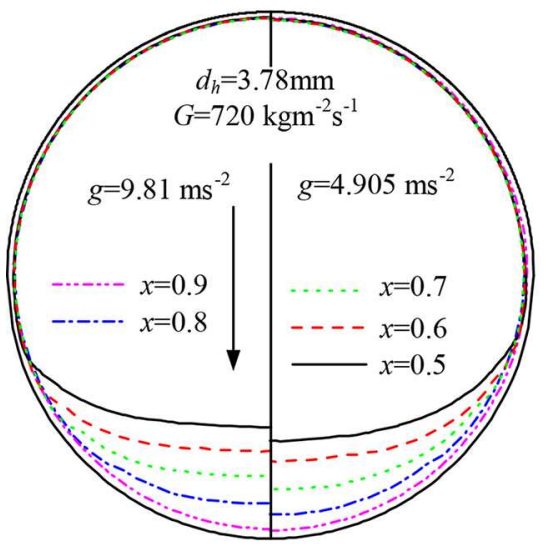

(c)

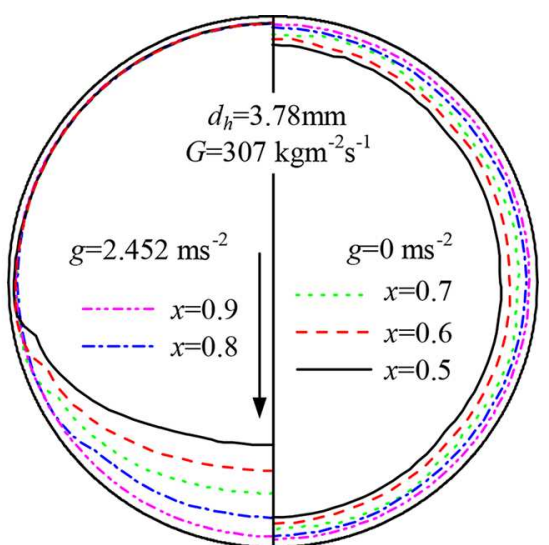

(b)

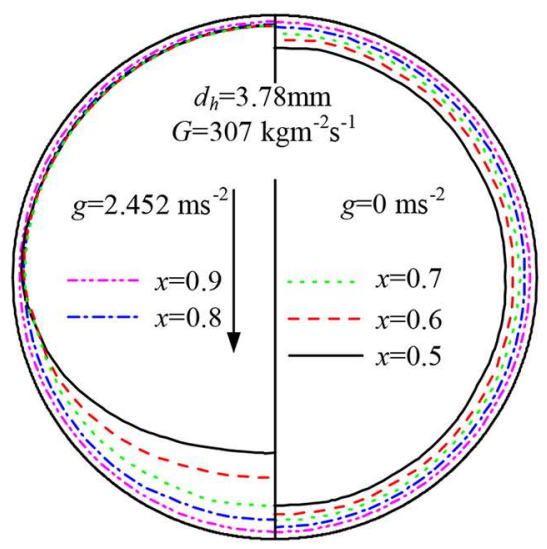

(d)

Figure 6. Liquid-vapor interfaces inside round tubes under different gravitational accelerations at $G=307$ and $720 \mathrm{~kg} \mathrm{~m}^{-2} \mathrm{~s}^{-1}$. 

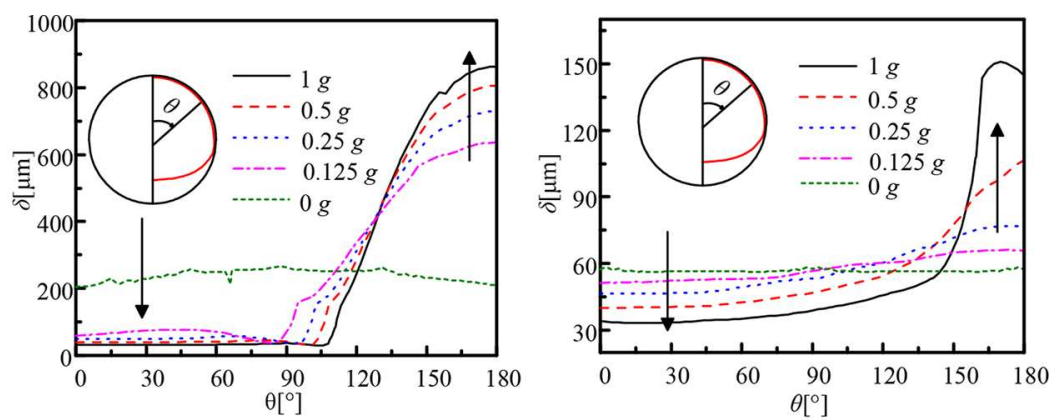

(a) $G=307 \mathrm{~kg} \mathrm{~m}^{-2} \mathrm{~s}^{-1}, x=0.5$

(b) $G=307 \mathrm{~kg} \mathrm{~m}^{-2} \mathrm{~s}^{-1}, x=0.9$

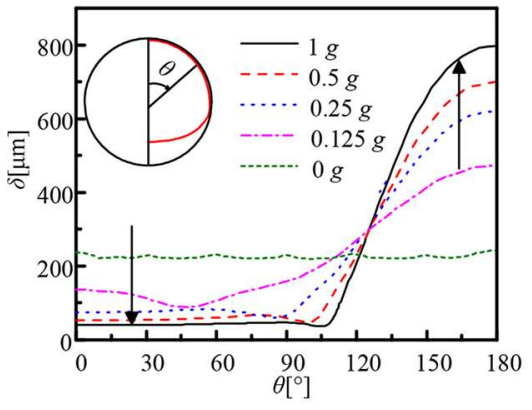

(c) $G=720 \mathrm{~kg} \mathrm{~m}^{-2} \mathrm{~s}^{-1}, x=0.5$

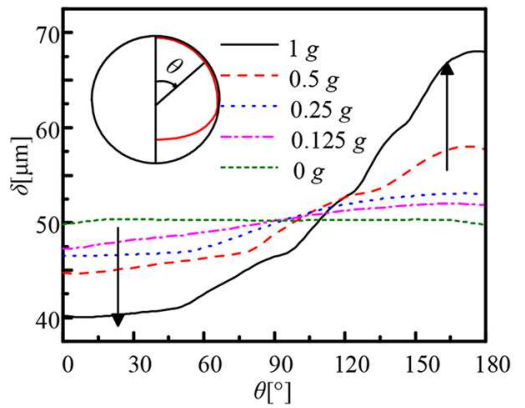

(d) $G=720 \mathrm{~kg} \mathrm{~m}^{-2} \mathrm{~s}^{-1}, x=0.9$

Figure 7. The liquid film thickness inside smooth tubes under different gravity accelerations at $G=307$ and $720 \mathrm{~kg} \mathrm{~m}^{-2} \mathrm{~s}^{-1}$.

\subsection{Liquid-vapor interfaces, liquid film thicknesses, and local heat transfer coefficients under reduced gravity}

For a better understanding of the condensation process under various gravities, the liquid-vapor interfaces are shown in Figure 6. The gravity effect tends to drive the liquid-vapor interface from the upper part to the tube bottom, leading to a thin liquid film at the top and a thicker liquid film at the bottom. The liquid film thickness at the top of the tubes remains nearly constant at each vapor quality except for the case of $0 \mathrm{~g}$, in which the liquid film at the top increases with vapor qualities, whereas the liquid film thickness at the bottom tends to decrease with decreasing gravity. When the gravity effect is neglected, a total axisymmetric annular flow is observed in Figure $6 b$ and $d$. At higher mass fluxes and vapor qualities, the shear force is more pronounced, and the liquid-vapor interfaces are nearly symmetrical for all cases.

Figure 7 illustrates a quantitative comparison of the local liquid film thickness, defined along the local orthogonal directions, versus the angular coordinate moving from the tube top to the bottom under different gravity at $x=0.9$ and $x=0.5$. For the cases of zero gravity, the liquid film thickness remains constant along the angular coordinate. The liquid film thickness decreases with increasing gravity at the top part of the tube for both vapor qualities, whereas the reverse is true at the bottom. The difference between the maximum and minimum liquid film thickness decreases with decreasing gravitational accelerations, which means that a more axisymmetric liquid-vapor interface will be formed at lower gravity. The liquid film thickness remains nearly constant from the tube top $\left(\theta=0^{\circ}\right)$ to $\theta=105^{\circ}$ under normal gravity at $x=0.5$, and it increases dramatically at $\theta>105^{\circ}$ because of the liquid accumulation due to the gravity effect. It should be noted that the minimum liquid film thickness is obtained at an angle close to $105^{\circ}$, whereas this angle decreases with decreasing gravity accelerations. The average liquid film thickness is nearly the same under different gravitational accelerations at the same vapor quality and mass flux. The average liquid film thicknesses are about $56 \mu \mathrm{m}$ at $x=0.9$ and $230 \mu \mathrm{m}$ at $\mathrm{x}=0.5$ at $G=307 \mathrm{~kg} \mathrm{~m}^{-2} \mathrm{~s}^{-1}$ for various gravities, whereas these values decrease to $50 \mu \mathrm{m}$ at $x=0.9$ and $215 \mu \mathrm{m}$ at $x=0.5$ when the mass flux is $720 \mathrm{~kg} \mathrm{~m}^{-2} \mathrm{~s}^{-1}$. 


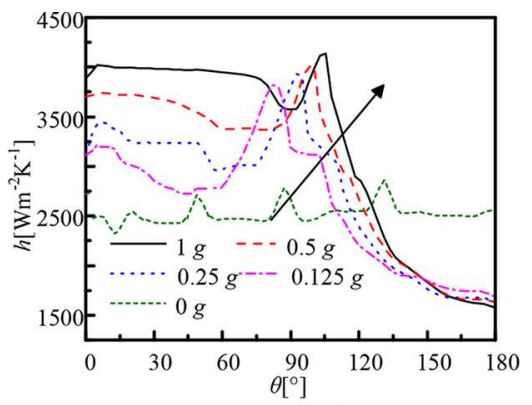

(a) $G=307 \mathrm{~kg} \mathrm{~m}^{-2} \mathrm{~s}^{-1}, x=0.5$

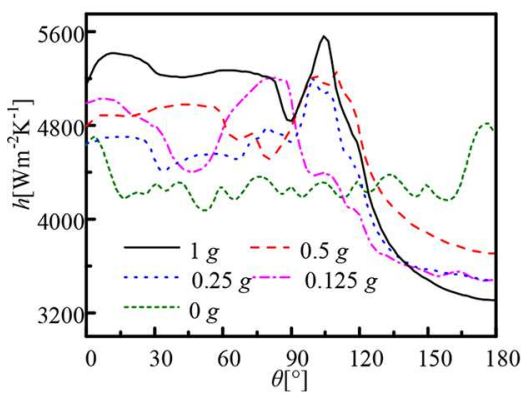

(c) $G=720 \mathrm{~kg} \mathrm{~m}^{-2} \mathrm{~s}^{-1}, x=0.5$

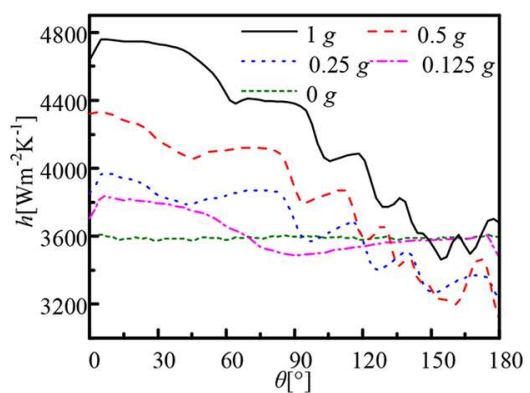

(b) $G=307 \mathrm{~kg} \mathrm{~m}^{-2} \mathrm{~s}^{-1}, x=0.9$

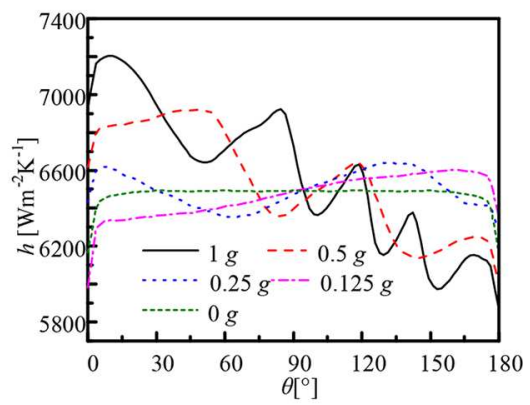

(d) $G=720 \mathrm{~kg} \mathrm{~m}^{-2} \mathrm{~s}^{-1}, x=0.9$

Figure 8. The local heat transfer coefficients for R410A inside smooth tubes under different gravity accelerations.

A quantitative comparison of the local heat transfer coefficient versus the angular coordinate under different gravity at $x=0.5$ and $x=0.9$ is shown in Figure 8. Opposite to the liquid film thickness, the local heat transfer coefficients increase with increasing gravity at the top of the tube and decrease with increases in gravity at the bottom. In the present work, a constant temperature boundary condition is applied at the tube wall, and the liquid-vapor interfacial temperature is within a small range of the saturation temperature. This means that a thin liquid film thickness will result in a larger temperature gradient, thus enhancing the heat transfer. Da Riva and Del Col [25] pointed out that the bottom part of the tube has a limited contribution to the global heat transfer coefficient for a stratified flow regime. The average heat transfer coefficient increases with increasing vapor quality and mass flux at a specific gravitational acceleration, and it also increases with increasing gravity at lower vapor quality and mass flux. The average heat transfer coefficient for normal gravity is about 1.3 times of that under zero gravity at $\mathrm{x}=0.5$ and $G=307 \mathrm{~kg} \mathrm{~m}^{-2} \mathrm{~s}^{-1}$. However, the difference between the average heat transfer coefficient obtained under varying gravity at $G=720 \mathrm{~kg} \mathrm{~m}^{-2} \mathrm{~s}^{-1}$ is unnoticeable compared with that at $G=307 \mathrm{~kg} \mathrm{~m}^{-2} \mathrm{~s}^{-1}$. With increasing mass flux, the shear force dominates the condensation process, whereas the gravity effect is unnoticeable at higher mass flux. Similar conclusions are also reported in the numerical work of Da Riva and Del Col [24] and the experimental study of Lee et al. [7]. For space missions involving multigravity, the influence of gravity can be negated by increasing mass flux. However, the increasing pressure drops should be considered when adopting such approaches.

\subsection{Stream traces under normal and zero gravity}

Stream traces at different vapor qualities, mass fluxes, and gravitational acceleration are shown in Figure 9. Only the $X$-axis and $Y$-axis components of velocity are considered here. The dashed red line presents $T=319.9 \mathrm{~K}$, and the solid red line stands for $T=319.5 \mathrm{~K}$. The liquid-vapor interfaces are blue solid lines. Obviously, the liquid-vapor interfaces locate within $319.5 \mathrm{~K}$ and $319.9 \mathrm{~K}$.

At $x=0.9$ and normal gravity, a vortex with its core lying at the tube bottom is observed for both mass fluxes, whereas this vortex is unnoticeable at $0 \mathrm{~g}$. The stream trace starts from the $Z$-axis and 


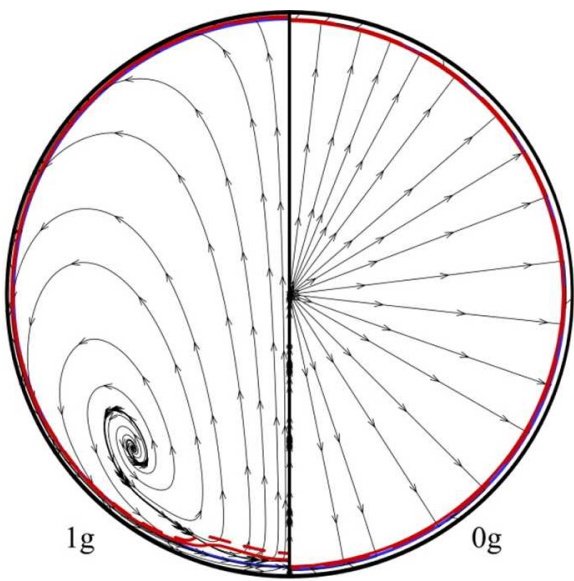

(a) $G=307 \mathrm{~kg} \mathrm{~m}^{-2} \mathrm{~s}^{-1}, x=0.9$

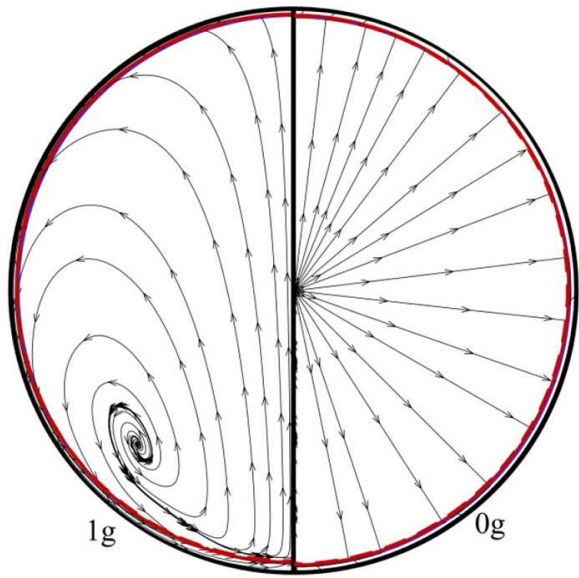

(c) $G=720 \mathrm{~kg} \mathrm{~m}^{-2} \mathrm{~s}^{-1}, x=0.9$

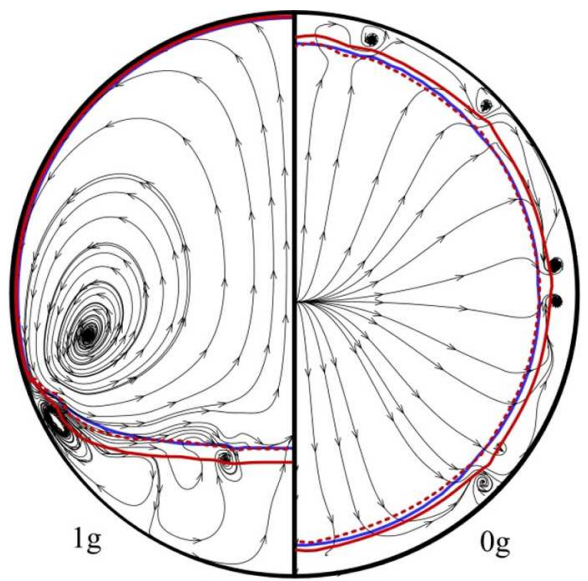

(b) $G=307 \mathrm{~kg} \mathrm{~m}^{-2} \mathrm{~s}^{-1}, x=0.5$

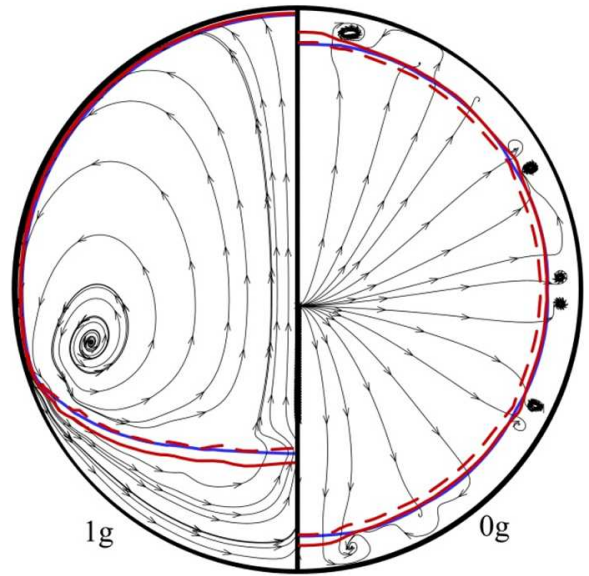

(d) $G=720 \mathrm{~kg} \mathrm{~m}^{-2} \mathrm{~s}^{-1}, x=0.5$

Figure 9. The stream traces in different cross sections under normal and zero gravity.

points to the liquid-vapor interface under zero gravity. As the vapor qualities decrease, the gravity effect is more pronounced. The height of the vortex center decreases with increasing mass flux and decreasing vapor quality. In the liquid film region, the refrigerant flows along the tube wall from the top to the bottom similar to the case of falling film, whereas the flow pattern in this region for the case of zero gravity is very different. Some small vertexes are observed in the liquid film region at zero gravity, which will disturb the flow and thermal boundary layers and lead to some peaks of the local heat transfer coefficients, as shown in Figure $8 a$.

For the condensing flow, the vapor phase turns into the liquid phase at the liquid-vapor interface, leading to a mass sink for the vapor phase [29]. As compensation for the mass sink, the vapor phase tends to flow to the liquid-vapor interfaces, as shown in Figure $9 b$ and $d$. Transportation of the vapor phase from the core region to the wall region will also enhance the heat transfer. As mentioned before, the gravity effect tends to pull the vapor and liquid phases to the bottom of the tube. The combined effect of gravity and the mass sink at the liquid-vapor interface is the reason for such stream traces for the condensation process under normal gravity.

\subsection{The mass transfer rate under normal and zero gravity}

Figure 10 shows the mass transfer rate from the vapor phase to the liquid phase at $x=0.5$ under normal and zero gravity. The mass transfer rate at zero gravity is much lower than that of normal 


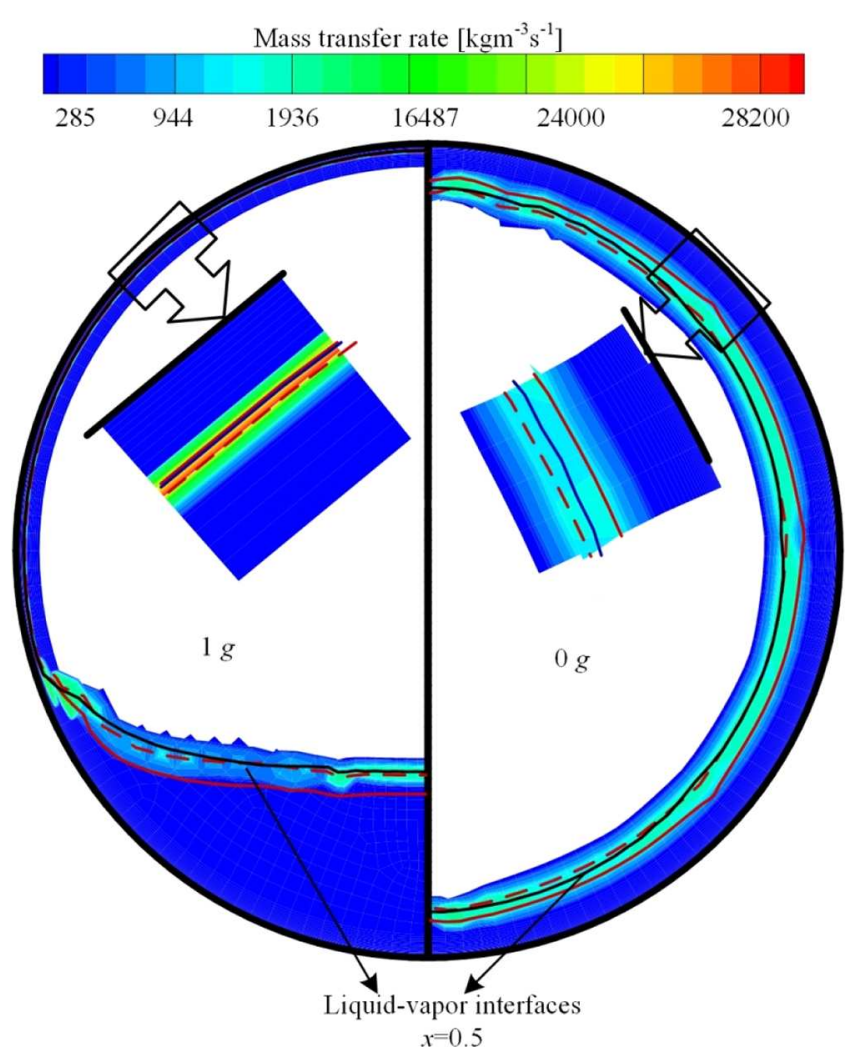

Figure 10. The mass transfer rate from vapor to liquid for R410A inside smooth tubes under different gravities.

gravity. Under normal gravity, the mass transfer rate at the tube bottom is much smaller than that at the tube top, which means the condensation process mainly occurs at the top of the tube. The liquid, which condenses at the upper part of the tube, is drained to the bottom by gravity instead of being carried in the tube axial direction by the shear stress. Under zero gravity, the mass transfer rate is nearly uniform along the perimeter direction, and the condensation process is mainly dominated by the shear force and surface tension. The mass transfer rate is directly related to the heat transfer coefficients in the condensation process under constant temperature boundary conditions through the latent heat calculated. With decreasing vapor quality, the liquid-vapor interface shrinks toward the core of the tube, resulting in a smaller heat transfer surface area, a lower mass transfer rate, and a lower heat transfer coefficient.

\section{Conclusions}

In this study, the heat transfer characteristics of condensation inside horizontal round tubes are numerically investigated for R410A under normal and reduced gravity. The following conclusions can be drawn from the present work.

1. The liquid film is dragged from the tube top to the bottom by gravity, leading to a very thin liquid film at the upper part. The liquid film thickness decreases with increasing gravity at the upper part of the tube, whereas the reverse is true at the bottom. The average liquid film thicknesses are about $56 \mu \mathrm{m}$ at $x=0.9$ and $230 \mu \mathrm{m}$ at $x=0.5$ at $\mathrm{G}=307 \mathrm{~kg} \mathrm{~m}^{-2} \mathrm{~s}^{-1}$ for various levels of gravity, whereas these value decrease to $50 \mu \mathrm{m}$ at $x=0.9$ and $215 \mu \mathrm{m}$ at $x=0.5$ when the mass flux is $720 \mathrm{~kg} \mathrm{~m}^{-2} \mathrm{~s}^{-1}$.

2. The local heat transfer coefficients increase with increasing gravity at the top of the tube and decrease with increases in gravity at the bottom. The bottom part of the tube has a limited 
contribution to the overall heat transfer coefficient when the liquid film accumulates at the bottom. The average heat transfer coefficients increase with increasing gravity accelerations at $G=307 \mathrm{~kg} \mathrm{~m}^{-2} \mathrm{~s}^{-1}$, whereas the difference of these under various gravity levels is unnoticeable at a higher mass flux. The proportion of the thin liquid film region is important for the overall heat transfer coefficients in the condensing flow.

3. A vortex with its core lying at the bottom of the tube is observed under normal gravity, whereas this vortex is unnoticeable at $0 \mathrm{~g}$. In the liquid film region, the refrigerant flows along the tube wall from the top to the bottom like the case of the falling film. The combined effect of gravity and the mass sink at the liquid-vapor interface is the reason for the stream traces found under normal gravity for the condensing flow. The mass transfer rate under zero gravity is much lower than that of normal gravity.

\section{Funding}

This work was supported by the National Science Foundation of Zhejiang Province, China (LZ13E060001), National Science Foundation of China (51210011), and the Zhejiang Provincial Public Projects (analysis and test) of Zhejiang Province (2015C37027).

\section{References}

[1] V. K. Dhir, and G. R. Warrier, E. Aktinol, Numerical Simulation of Pool Boiling: A Review, J. Heat Transfer, vol. 135, pp. 061502-061502, 2013.

[2] J. Kim, Review of Nucleate Pool Boiling Bubble Heat Transfer Mechanisms, Int. J. Multiphase Flow, vol. 35, pp. 1067-1076, 2009.

[3] J. Straub, Boiling Heat Transfer and Bubble Dynamics in Microgravity, Advances in Heat Transfer, Vol. 35, pp. $57-172,2001$.

[4] J. F. Zhao, Two-Phase Flow and Pool Boiling Heat Transfer in Microgravity, Int. J. Multiphase Flow, vol. 36, pp. 135-143, 2010.

[5] C. Konishi and I. Mudawar, Review of Flow Boiling and Critical Heat Flux in Microgravity, Int. J. Heat Mass Transfer, vol. 80, pp. 469-493, 2015.

[6] H. Lee, I. Park, C. Konishi, I. Mudawar, R. I. May, J. R. Juergens, J. D. Wagner, N. R. Hall, H. K. Nahra, M. M. Hasan, and J. R. Mackey, Experimental Investigation of Flow Condensation in Microgravity, J. Heat Transfer, vol. 136, pp. 11, 2014.

[7] H. Lee, I. Mudawar, and M. M. Hasan, Experimental and Theoretical Investigation of Annular Flow Condensation in Microgravity, Int. J. Heat Mass Transfer, vol. 61, pp. 293-309, 2013.

[8] S. Bortolin, G. El Achkar, M. Kostoglou, A. Glushchuk, T. D. Karapantsios, P. Lavieille, D. Del Col, C. Buffone, M. Miscevic, B. Toth, and O. Minster, Experimental Investigations on Condensation in the Framework of ENhanced COndensers in Microgravity (ENCOM-2) Project, Microgravity Sci. Tec., vol. 26, pp. 335-349, 2014.

[9] S. Lips and J. P. Meyer, Two-phase Flow in Inclined Tubes with Specific Reference to Condensation: A Review, Int. J. Multiphase Flow, vol. 37, pp. 845-859, 2011.

[10] M. Akhavan-Behabadi, R. Kumar, and S. Mohseni, Condensation Heat Transfer of R-134a Inside a Microfin Tube with Different Tube Inclinations, Int. J. Heat Mass Transfer, vol. 50, pp. 4864-4871, 2007.

[11] S. G. Mohseni and M. A. Akhavan-Behabadi, Visual Study of Flow Patterns During Condensation Inside a Microfin Tube with Different Tube Inclinations, Int. Commun. Heat Mass, vol. 38, pp. 1156-1161, 2011.

[12] S. Lips and J. P. Meyer, Experimental Study of Convective Condensation in An Inclined Smooth Tube. Part II: Inclination Effect on Pressure Drops and Void Fractions, Int. J. Heat Mass Transfer, vol. 55, pp. 405-412, 2012.

[13] S. Lips and J. P. Meyer, Experimental Study of Convective Condensation in An Inclined Smooth Tube. Part I: Inclination Effect on Flow Pattern and Heat Transfer Coefficient, Int. J. Heat Mass Transfer, vol. 55, pp. 395-404, 2012.

[14] D. Del Col, M. Bortolato, M. Azzolin, and S. Bortolin, Effect of Inclination During Condensation Inside a Square Cross Section Minichannel, Int. J. Heat Mass Transfer, vol. 78, pp. 760-777, 2014.

[15] H. Ganapathy, A. Shooshtari, K. Choo, S. Dessiatoun, M. Alshehhi, and M. Ohadi, Volume of Fluid-based Numerical Modeling of Condensation Heat Transfer and Fluid Flow Characteristics in Microchannels, Int. J. Heat Mass Transfer, vol. 65, pp. 62-72, 2013.

[16] H. Lee, C. R. Kharangate, N. Mascarenhas, I. Park, and I. Mudawar, Experimental and Computational Investigation of Vertical Downflow Condensation, Int. J. Heat Mass Transfer, vol. 85, pp. 865-879, 2015. 
[17] S. Chen, Z. Yang, Y. Duan, Y. Chen, and D. Wu, Simulation of Condensation Flow in a Rectangular Microchannel, Chem. Eng. Process. vol. 76, pp. 60-69, 2014.

[18] H. S. Wang and J. W. Rose, Heat Transfer and Pressure Drop During Laminar Annular Flow Condensation in Micro-Channels, Exp. Heat Transfer, vol. 26, pp. 247-265, 2013.

[19] H. S. Wang and J. W. Rose, Theory of Heat Transfer During Condensation in Microchannels, Int. J. Heat Mass Transfer, vol. 54, pp. 2525-2534, 2011.

[20] H. S. Wang and J. W. Rose, Surface Tension-affected Laminar Film Condensation Problems, J. Mech. Sci. Technol., vol. 21, pp. 1760-1774, 2007.

[21] H. S. Wang and J. W. Rose, Film Condensation in Microchannels: Effect of Tube Inclination, in Proceedings of the 4th International Conference on Nanochannels, Microchannnels, and Minichannels, Pts A and B, Amer Soc Mechanical Engineers, Limerick, 2006, pp. 133-137.

[22] H. S. Wang and J. W. Rose, Film Condensation in Horizontal Microchannels: Effect of Channel Shape, Int. J. Therm. Sci., vol. 45, pp. 1205-1212, 2006.

[23] H. S. Wang and J. W. Rose, A Theory of Film Condensation in Horizontal Noncircular Section Microchannels, J. Heat Transfer, vol. 127, pp. 1096-1105, 2005.

[24] E. Da Riva and D. Del Col, Effect of Gravity During Condensation of R134a in a Circular Minichannel, Microgravity Sci. Technol., vol. 23, pp. 87-97, 2011.

[25] E. Da Riva and D. Del Col, Numerical Simulation of Laminar Liquid Film Condensation in a Horizontal Circular Minichannel, J. Heat Transfer, vol. 134, pp. 051019-051019, 2012.

[26] E. Da Riva, D. Del Col, S. V. Garimella, and A. Cavallini, The Importance of Turbulence During Condensation in a Horizontal Circular Minichannel, Int. J. Heat Mass Transfer, vol. 55, pp. 3470-3481, 2012.

[27] S. Bortolin, E. Da Riva, and D. Del Col, Condensation in a Square Minichannel: Application of the Vof Method, Heat Transfer. Eng., vol. 35, pp. 193-203, 2014.

[28] J. Zhang, W. Li, and S. A. Sherif, A Numerical Study of Condensation Heat Transfer and Pressure Drop in Horizontal Round and Flattened Minichannels, Int. J. Therm. Sci., vol. 106, pp. 80-93, 2016.

[29] J. Zhang, W. Li, and W. J. Minkowycz, Numerical Simulation of Condensation for R410A at Varying Saturation Temperatures in Mini/micro Tubes, Numer. Heat Transfer, Part A, vol. 69, pp. 464-478, 2016.

[30] C. R. Kharangate, H. Lee, I. Park, and I. Mudawar, Experimental and Computational Investigation of Vertical Upflow Condensation in a Circular Tube, Int. J. Heat Mass Transfer, vol. 95, pp. 249-263, 2016.

[31] J. Brackbill, D. B. Kothe, and C. Zemach, A Continuum Method for Modeling Surface Tension, J. Comput. Phys., vol. 100, pp. 335-354, 1992.

[32] W. H. Lee, A Pressure Iteration Scheme for Two-phase Flow Modeling, Hemisphere, Washington, DC, 1980.

[33] J. Wei, L. Pan, D. Chen, H. Zhang, J. Xu, and Y. Huang, Numerical Simulation of Bubble Behaviors in Subcooled Flow Boiling under Swing Motion, Nucl. Eng. Des., vol. 241, pp. 2898-2908, 2011.

[34] Z. Yang, X. Peng, and P. Ye, Numerical and Experimental Investigation of Two Phase Flow During Boiling in a Coiled Tube, Int. J. Heat Mass Transfer, vol. 51, pp. 1003-1016, 2008.

[35] Z. Liu, B. Sunden, and J. Yuan, VOF Modeling and Analysis of Filmwise Condensation Between Vertical Parallel Plates, Heat Transfer Res., vol. 43, pp. 43-49, 2012.

[36] M. K. Dobson and J. C. Chato, Condensation in Smooth Horizontal Tubes, J. Heat Transfer, vol. 120, pp. 193-213, 1998.

[37] A. Cavallini, G. Censi, D. Del Col, and L. Doretti, In-tube Condensation of Halogenated Refrigerants, ASHRAE Trans., vol. 108, pp. 146, 2002.

[38] H. M. Soliman, On the Annular-to-wavy Flow Pattern Transition During Condensation Inside Horizontal Tubes, Can. J. Chem. Eng., vol. 60, pp. 475-481, 1982.

[39] A. Cavallini, D. Del Col, L. Doretti, M. Matkovic, L. Rossetto, C. Zilio, and G. Censi, Condensation in Horizontal Smooth Tubes: A New Heat Transfer Model for Heat Exchanger Design, Heat Transf. Eng., vol. 27, pp. 31-38, 2006.

[40] Z. Wu, B. Sundén, L. Wang, and W. Li, Convective Condensation Inside Horizontal Smooth and Microfin Tubes, J. Heat Transfer, vol. 136, pp. 051504-051504, 2014.

[41] J. R. Thome, J. El Hajal, and A. Cavallini, Condensation in Horizontal Tubes, Part 2: New Heat Transfer Model Based on Flow Regimes, Int. J. Heat Mass Transfer, vol. 46, pp. 3365-3387, 2003.

[42] M. M. Shah, An Improved and Extended General Correlation for Heat Transfer During Condensation in Plain Tubes, HVAC\&R Res., vol. 15, pp. 889-913, 2009. 